\title{
HALLAZGOS TOMOGRÁFICOS PULMONARES ASOCIADOS A SEVERIDAD Y MORTALIDAD EN PACIENTES CON LA COVID-19
}

\author{
Jheferson Contreras-Grande (ib),a, Vanessa Pineda-Borja@1,2,b, Hubertino Díaz(11,2,c, \\ Renzo J.C. Calderon-Anyosa ${ }^{2, d}$, Bertha Rodríguez ${ }^{1, a}$, María Morón (1,a. \\ ${ }^{1}$ Departamento de Imagenología, Hospital Nacional Edgardo Rebagliati Martins, Lima, Perú. \\ 2 Facultad de Medicina «Alberto Hurtado», Universidad Peruana Cayetano Heredia, Lima, Perú. \\ a Médico especialista en Radiología; ${ }^{b}$ médica residente de Radiología; ' médico especialista en Radiología, maestro en \\ Medicina; ${ }^{\mathrm{d}}$ médico cirujano, maestro en Informática Biomédica en Salud Global.
}

\section{RESUMEN}

Objetivos: Determinar los hallazgos tomográficos pulmonares asociados a severidad y mortalidad en pacientes con la COVID-19 del Hospital Nacional Edgardo Rebagliati Martins (HNERM), y proponer puntos de corte para una puntuación tomográfica de severidad (PTS). Materiales y métodos: Estudio de cohorte retrospectivo en 254 pacientes con la COVID-19 que contaban con tomografía de tórax clasificada según severidad clínica. Se realizó un análisis multivariado mediante regresión logística para determinar asociación con la severidad clínica, un análisis de regresión de Cox para evaluar mortalidad, y curvas ROC para evaluar la PTS. Resultados: Los hallazgos tomográficos asociados a severidad clínica fueron el patrón difuso de las lesiones (OR: 3,23, IC 95\%: 1,46-7,14), patrón en «empedrado» (OR: 2,48; IC 95\%: 1,08-5,68) y mayor valor en la PTS (OR: 1,73; IC 95\%: 1,49-2,02). Los hallazgos asociados a mortalidad fueron el patrón en «empedrado» (HR: 1,78; IC 95\%: 1,03-3,06) y mayor valor en la PTS (HR: 1,33; IC 95\%: 1,20-1,48). Un valor de 7 en la PTS tuvo una sensibilidad de $94,4 \%$ y especificidad de $100 \%$ para identificar casos moderados y un valor de 13 tuvo una sensibilidad de $84,9 \%$ y una especificidad de 70,6\% para casos severos. Conclusiones: El patrón difuso de las lesiones se asoció a una mayor severidad clínica. El patrón en «empedrado» y un mayor valor en la PTS se asociaron a mayor severidad clínica y a mortalidad. Se proponen los valores de 7 y 13 como puntos de corte de la PTS para identificar casos moderados y severos.

Palabras clave: COVID-19; SARS-CoV-2; Neumonía; Tomografía; Índice de Severidad de Enfermedad; Mortalidad (fuente: DeCS BIREME).

\section{CHEST COMPUTED TOMOGRAPHY FINDINGS ASSOCIATED WITH SEVERITY AND MORTALITY IN PATIENTS WITH COVID-19}

Citar como: Contreras-Grande J, Pineda-Borja V, Díaz H, CalderonAnyosa RJC, Rodríguez B, Morón M. Hallazgos tomográficos pulmonares asociados a severidad y mortalidad en pacientes con la COVID-19. Rev Peru Med Exp Salud Publica. 2021;38(2):20613. doi: https://doi.org/10.17843/ rpmesp.2021.382.6562.

Correspondencia: Vanessa Pineda Borja; Av. Juan Pardo de Zela 642, Lince. Lima, Perú; vanessa.pineda@upch.pe

Recibido: $12 / 10 / 2020$ Aprobado: 03/03/2021 En línea: 05/04/2021

\section{ABSTRACT}

Objectives: To determine chest CT findings associated with severity and mortality in patients with COVID-19 from the Hospital Nacional Edgardo Rebagliati Martins (HNERM) and propose cut-off values for a tomographic severity score (TSS). Materials and Methods: A retrospective cohort study was conducted in 254 patients with COVID-19 who underwent chest CT as part of their initial evaluation at the emergency room; they were classified according to clinical severity. Main tomographic findings were described. A multivariate analysis with logistic regression was carried out to determine association with clinical severity, the Cox model was used to evaluate mortality, and ROC curves were elaborated to assess cutoff values for the TSS. Results: CT findings associated with clinical severity were the following: diffuse pattern (OR: 3.23, 95\% CI: 1.46-7.14), crazy-paving pattern (OR: 2.48; 95\% CI: 1.08-5.68), and high TSS value (OR: 1.73; 95\% CI: 1.49-2.02). The crazy-paving pattern (HR: 1.78; 95\% CI: 1.03-3.06) and a high TSS value (HR: 1.33 ; 95\% CI: $1.20-1.48$ ) were found to be associated with mortality. A value of 7 in the TSS showed a sensibility of $94.4 \%$ and a specificity of $100 \%$ for moderate disease, and a value of 13 showed a sensibility of $84.9 \%$ and a specificity of $70.6 \%$ for severe disease. Conclusions: The diffuse pattern is associated with higher clinical severity. The crazy-paving pattern and a high TSS value are associated with higher clinical severity and mortality. We propose TSS cutoff values of 7 and 13 for moderate and severe disease, respectively.

Keywords: COVID-19; SARS-CoV-2; Pneumonia; Tomography; Severity of Illness Index; Mortality (source: MeSH NLM). 


\section{INTRODUCCIÓN}

En diciembre del 2019 el nuevo coronavirus del síndrome respiratorio agudo grave (de tipo) 2 (SARS-CoV-2) produjo en Wuhan, China, el primer brote de enfermedad neumónica conocida como COVID-19, la cual se convirtió en una pandemia en marzo del $2020^{(1-4)}$.

La tomografía computarizada (TC) de tórax ha demostrado ser útil en la evaluación de pacientes con enfermedad moderada y severa, así como en aquellos que presentan deterioro de la función respiratoria. Debido a ello, la sociedad internacional de Fleischner ${ }^{(5)}$ recomienda su uso en este grupo de pacientes y por el contrario, no las recomienda como prueba de tamizaje en pacientes asintomáticos, ni como evaluación de pacientes con enfermedad leve.

Múltiples estudios ${ }^{(6-10)}$ han evaluado el rendimiento diagnóstico de la TC en comparación con la prueba de referencia (reacción en cadena de la polimerasa con transcriptasa reversa [RT-PCR]) y han reportado una sensibilidad y especificidad combinada de $94 \%$ y $37 \%$, respectivamente ${ }^{(11)}$. Los principales hallazgos tomográficos ${ }^{(12-15)}$ son la presencia de opacidades en vidrio esmerilado, consolidaciones y patrón en «empedrado» de distribución periférica bilateral; mientras que la presencia de efusión pleural y adenopatías han sido reportadas con poca frecuencia.

Otros estudios ${ }^{(16-20)}$ han evaluado la relación entre los hallazgos tomográficos y el grado de severidad clínica, así como el riesgo de muerte y han propuesto distintas puntuaciones tomográficas para valorar la extensión del compromiso pulmonar. Entre ellas, la puntuación tomográfica de severidad (PTS), propuesta por Li et al. ${ }^{(16)}$, resulta la más práctica y factible de aplicar, sin embargo, el autor sugiere un único punto de corte de 7,5 para determinar enfermedad severa-crítica. Este modelo no se adecúa a la clasificación de severidad clínica (leve, moderado y severo) que se aplica a nuestros pacientes ${ }^{(21)}$, por lo que resulta importante validar esta puntuación tomográfica en nuestro país.

El objetivo de este estudio es determinar los hallazgos tomográficos pulmonares asociados a severidad y mortalidad en pacientes con la COVID-19 atendidos en el Hospital Nacional Edgardo Rebagliati Martins (HNERM) de Lima, Perú, y proponer puntos de corte para la PTS.

\section{MATERIALES Y MÉTODOS}

\section{Población, diseño de estudio y recolección de datos}

Se realizó un estudio de cohorte retrospectivo. Se incluyó a todos los pacientes mayores de 18 años atendidos entre el 1 y 30 de abril del 2020 en la emergencia del HNERM en Lima, Perú, que fueron casos confirmados de la COVID-19

\section{MENSAJES CLAVE}

Motivación para realizar el estudio: Se desconocen los hallazgos tomográficos relacionados a mayor severidad y mortalidad en pacientes con la COVID-19 en el Perú.

Principales hallazgos: El patrón difuso de las lesiones se asoció a mayor severidad clínica. El patrón en «empedrado» y un mayor valor en la PTS se asociaron a mayor severidad clínica y a mortalidad. Se proponen valores de 7 y 13 como puntos de corte de la PTS para identificar casos moderados y severos.

Implicancias: Los resultados de este estudio ayudarían a mejorar la evaluación clínica de los pacientes con la COVID-19 en el ámbito hospitalario.

y a quienes se les solicitó TC de tórax durante su evaluación inicial.

Se utilizó la definición operacional del Ministerio de Salud (MINSA) ${ }^{(21)}$ y se consideraron confirmados a aquellos casos sospechosos que tuvieron una prueba molecular o serológica positiva para infección por SARS-CoV-2 realizada en el hospital. Se excluyeron a pacientes que tuvieron datos incompletos en su historia clínica o una TC con artefactos importantes que limitaron su evaluación. Así, de un total de 309 pacientes con diagnóstico confirmado y TC de tórax, se excluyeron a 53 que tenían datos incompletos y a dos con artefactos importantes, lo que limitó el número a 254 pacientes (Anexo 1).

De la historia clínica electrónica se recolectaron variables demográficas y clínicas, como edad en años ( $>60$ años, $\leq 60$ años), sexo (femenino, masculino), severidad clínica (leve, moderada, severa), tiempo de enfermedad en días, síntomas (disnea, fiebre $\geq 38^{\circ} \mathrm{C}$, tos, malestar general, dolor de garganta, diarrea), comorbilidades (hipertensión arterial, diabetes mellitus (DM), obesidad, enfermedad renal crónica, asma) y tiempo de hospitalización en días.

Se recolectaron datos de laboratorio, como leucocitos $\left(\mathrm{x} 10^{3} / \mathrm{uL}\right)$, linfocitos $\left(\mathrm{x} 10^{3} / \mathrm{uL}\right)$, aspartato aminotransferasa (AST) $(\mathrm{U} / \mathrm{L})$, alanina aminotransferasa (ALT) (U/L), deshidrogenasa láctica $(\mathrm{DHL})(\mathrm{U} / \mathrm{L})$, tiempo de protrombina (TP) (s), tiempo de tromboplastina parcial activada (TTP) $(\mathrm{s})$, creatinina $(\mathrm{mg} / \mathrm{dL})$, proteína $\mathrm{C}$ reactiva $(\mathrm{mg} / \mathrm{dL})$, ferritina sérica $(\mathrm{ng} / \mathrm{mL})$ y dímero $\mathrm{D}(\mathrm{ug} / \mathrm{mL})$.

La severidad clínica se determinó de acuerdo a los lineamientos establecidos por el MINSA ${ }^{(21)}$ : caso leve, si tenía al menos dos signos o síntomas de la COVID-19; caso moderado, si tenía al menos un criterio de hospitalización (disnea, frecuencia respiratoria $(\mathrm{FR})>22$ respiraciones por minuto (RPM), saturación de oxígeno $<95 \%$, alteración del nivel de consciencia, hipotensión, signos de neumonía, recuento lin- 
focitario $<1000$ células/ $\mu \mathrm{L}$ ) y caso severo si tenía signos de sepsis y/o falla respiratoria (al menos dos de FR $>22 \mathrm{RPM}$, $\mathrm{PaCO}_{2}<32 \mathrm{mmHg}$, alteración del nivel de consciencia, hipotensión, $\mathrm{PaO}_{2}<60 \mathrm{mmHg}$ o $\mathrm{PaFi}<300$, signos clínicos de fatiga muscular, lactato sérico $>2 \mathrm{mosm} / \mathrm{L}$ ).

\section{Adquisición y análisis de las imágenes}

Las TC de tórax fueron tomadas en el hospital con el paciente en decúbito supino y sin la administración de material de contraste, en un tomógrafo Toshiba Aquilion Prime de 80 filas de detectores con sistema multicorte.

El análisis de las imágenes fue realizado por dos médicos radiólogos con 3 y 21 años de experiencia (JCG y MM), quienes utilizaron los términos tomográficos listados en el glosario de la Sociedad de Fleischner ${ }^{(22)}$. Se registró la presencia de los siguientes hallazgos tomográficos: opacidades en vidrio esmerilado, consolidaciones, patrón en «empedrado», engrosamiento intersticial interlobulillar (EII), signo del «halo reverso», bandas parenquimales, broncograma aéreo, efusión pleural, adenopatía (linfonodo mediastinal con eje corto $>10$ $\mathrm{mm}$ ) y el signo «preservación subpleural» (región de 1-2 mm de parénquima pulmonar subpleural no opacificado). Se registró también la distribución de las lesiones (periférica, central o mixta), el patrón de estas (en parches o difuso), así como los lóbulos pulmonares comprometidos y el número de ellos. Se calculó el valor de la PTS asignando un valor de entre 0 y 4 a cada uno de los cinco lóbulos pulmonares según su grado de compromiso: 0 (0\%), 1 (1-25\%), 2 (26-50\%), 3 (51-75\%) y 4 (76-100\%), siendo la suma de estos valores la puntuación final, con valores de entre 0 y $20^{(16)}$.

\section{Análisis estadístico}

Las variables cuantitativas se expresaron como media y desviación estándar $(\mathrm{DE})$ o como mediana y rango intercuartílico (RIC), mientras que las variables categóricas se expresaron como porcentajes.

Para determinar diferencias entre sobrevivientes y fallecidos se utilizó la prueba exacta de Fisher o la prueba de Chi cuadrado para variables cualitativas. Se evaluó la normalidad de las variables cuantitativas mediante histogramas y la prueba de Jarque-Bera. Si la distribución era normal, se utilizó la prueba de T de Student, de lo contrario, se utilizó la prueba de suma de rangos de Wilcoxon.

Se calculó un coeficiente de correlación intraclase (CCI) para evaluar la consistencia entre ambos observadores en la valoración de la PTS. Asimismo, se elaboraron curvas ROC para determinar los puntos de corte que tuvieran la combinación de sensibilidad y especificidad más alta para identificar casos moderados y casos severos.

Los hallazgos tomográficos asociados a la severidad clínica fueron evaluados utilizando un modelo de regresión logística multivariada, se agruparon las categorías leve y mo- derada y se compararon con la categoría severa. El modelo se ajustó con variables como edad, sexo, tiempo de enfermedad y comorbilidades, como hipertensión arterial (HTA), obesidad y diabetes mellitus, por tratarse de factores de riesgo reconocidos en casos de severidad y mortalidad en pacientes con la COVID-19 ${ }^{(23-26)}$.

Para determinar los hallazgos tomográficos asociados a mortalidad en pacientes con enfermedad grave, se realizó un análisis de regresión de Cox multivariado ajustado para las mismas variables del modelo logístico, obteniéndose hazard ratios (HR) para mortalidad. Se comprobó la proporcionalidad de los HR de cada parámetro y del modelo global mediante el gráfico de los residuos de Schoenfeld y la prueba de pendiente distinta de cero. El tiempo de sobrevida en días se contabilizó desde el día de la admisión del paciente hasta el evento muerte, alta hospitalaria o final del periodo de seguimiento (86 días). El análisis estadístico fue realizado utilizando el programa Stata versión 16.1.

\section{Aspectos éticos}

El estudio fue aprobado por el Comité de Ética en Investigación Específico para COVID-19 de EsSALUD y por el Comité Institucional de Ética en Investigación (CIEI) de la Universidad Peruana Cayetano Heredia (constancia E086 - 16 - 20) bajo la categoría de exento de revisión. No se requirió la aplicación de un formato de consentimiento informado ya que el estudio solo incluyó revisión de historias clínicas y estudios tomográficos, con lo cual se preservó la confidencialidad de los pacientes.

\section{RESULTADOS}

\section{Características demográficas, clínicas y laboratoriales}

De los 254 pacientes incluidos en el estudio, 137 (53,9\%) tenían RT-PCR positivo y $117(46,1 \%)$ serología positiva. 173 $(68,1 \%)$ fueron varones y $81(31,9 \%)$ mujeres, la mediana de edad fue 59,1 (DE: 14,7 años). Con relación a la severidad clínica, 13 (5,1\%) pacientes fueron casos leves, 89 (35,0\%) moderados y $152(59,9 \%)$ severos. Al final del periodo de seguimiento, $158(62,2 \%)$ pacientes fueron dados de alta, 95 $(37,4 \%)$ fallecieron y $1(0,4 \%)$ permaneció en la unidad de cuidados intensivos (UCI). El resto de las variables demográficas y clínicas se muestran en la Tabla 1.

Las variables que mostraron diferencia significativa entre sobrevivientes y fallecidos fueron: edad, disnea, fiebre, diarrea, HTA y tiempo de hospitalización (Tabla 1). Los análisis de laboratorio evaluados al ingreso que mostraron diferencia significativa entre sobrevivientes y fallecidos fueron: leucocitos, linfocitos, DHL, TP, TTP, creatinina, proteína C reactiva, ferritina sérica y dímero $\mathrm{D}$ (Anexo 2 ). 
Tabla 1. Características demográficas y clínicas de pacientes con la COVID-19 atendidos en el Hospital Nacional Edgardo Rebagliati Martins, abril del 2020.

\begin{tabular}{|c|c|c|c|c|}
\hline \multirow[t]{2}{*}{ Características } & $\begin{array}{c}\text { Total } \\
\text { (254 casos) } \\
\end{array}$ & $\begin{array}{l}\text { Fallecidos } \\
(95 \text { casos }) \\
\end{array}$ & $\begin{array}{c}\text { Sobrevivientes } \\
\text { (159 casos) }\end{array}$ & \multirow[t]{2}{*}{ Valor de p } \\
\hline & $\mathrm{n}(\%)$ & $\mathrm{n}(\%)$ & $\mathrm{n}(\%)$ & \\
\hline Edad (años), media (DE) & $59,1(14,7)$ & $66,7(12,2)$ & $54,6(14,2)$ & $<0,001^{\text {a }}$ \\
\hline$>60$ & $125(49,2)$ & $72(75,8)$ & $53(33,3)$ & $<0,001^{\mathbf{b}}$ \\
\hline$\leq 60$ & $129(50,8)$ & $23(24,2)$ & $106(66,7)$ & \\
\hline Sexo & & & & $0,232^{\mathrm{b}}$ \\
\hline Masculino & $173(68,1)$ & $69(72,6)$ & $104(65,4)$ & \\
\hline Femenino & $81(31,9)$ & $26(27,4)$ & $55(34,6)$ & \\
\hline Severidad clínica & & & & NC \\
\hline Leve & $13(5,1)$ & 0 & $13(8,2)$ & \\
\hline Moderada & $89(35,0)$ & $1(1,1)$ & $88(55,3)$ & \\
\hline Severa & $152(59,9)$ & $94(98,9)$ & $58(36,5)$ & \\
\hline Tiempo de enfermedad (días), media (DE) & $10,2(5,2)$ & $10,3(5,2)$ & $10,1(5,3)$ & $0,622^{a}$ \\
\hline \multicolumn{5}{|l|}{ Síntomas } \\
\hline Disnea & $229(90,2)$ & $93(97,9)$ & $136(85,5)$ & $0,001^{\mathbf{b}}$ \\
\hline Fiebre $\geq 38^{\circ} \mathrm{C}$ & $215(84,7)$ & $73(76,8)$ & $142(89,3)$ & $0,008^{b}$ \\
\hline Tos & $215(84,7)$ & $79(83,2)$ & $136(85,5)$ & $0,611^{b}$ \\
\hline Malestar general & $167(65,8)$ & $63(66,3)$ & $104(65,4)$ & $0,883^{b}$ \\
\hline Dolor de garganta & $67(26,4)$ & $28(29,5)$ & $39(24,5)$ & $0,387^{\mathbf{b}}$ \\
\hline Diarrea & $43(16,9)$ & $9(9,5)$ & $34(21,4)$ & $0,014^{\mathrm{b}}$ \\
\hline \multicolumn{5}{|l|}{ Comorbilidades } \\
\hline HTA & $79(31,1)$ & $39(41,1)$ & $40(25,2)$ & $0,008^{b}$ \\
\hline Diabetes mellitus & $47(18,5)$ & $22(23,2)$ & $25(15,7)$ & $0,140^{\mathrm{b}}$ \\
\hline Obesidad & $40(15,8)$ & $19(20,0)$ & $21(13,2)$ & $0,150^{\mathrm{b}}$ \\
\hline ERC & $13(5,1)$ & $8(8,4)$ & $5(3,1)$ & $<0,001^{\mathrm{c}}$ \\
\hline Asma & $11(4,3)$ & $2(2,1)$ & $9(5,7)$ & $0,218^{c}$ \\
\hline Tiempo de hospitalización (días), mediana (RIC) & $11(6-20)$ & $7(4-12)$ & $13(8-23)$ & $<0,001^{\mathrm{d}}$ \\
\hline
\end{tabular}

DE: desviación estándar, RIC: rango intercuartílico, HTA: hipertensión arterial, ERC: enfermedad renal crónica, NC: no calculado.

${ }^{\text {a }}$ Prueba de T de Student, ${ }^{\mathrm{b}}$ prueba de Chi cuadrado, ${ }^{\mathrm{c}}$ prueba exacta de Fisher, ${ }^{\mathrm{d}}$ prueba de suma de rangos de Wilcoxon.

\section{Hallazgos tomográficos}

De las 254 TC de tórax evaluadas, 243 (95,7\%) presentaron opacidades en vidrio esmerilado, 200 (78,7\%) consolidaciones, 184 $(72,4 \%)$ «preservación subpleural», 182 (71,7\%) EII, 120 (47,2\%) broncograma aéreo, $90(35,4 \%)$ patrón en «empedrado» y 47 $(18,5 \%)$ bandas parenquimales. $15(5,9 \%)$ pacientes presentaron adenopatías, 7 (2,8\%) efusión pleural y 5 (2,0\%) el signo del «halo reverso». Las lesiones pulmonares presentaron con mayor frecuencia una distribución mixta $(78,3 \%)$, así como un patrón difuso (74\%), siendo menos frecuente el patrón en parches (26\%).

El signo «preservación subpleural», EII, patrón en «empedrado», adenopatías, distribución mixta y patrón difuso de las lesiones fueron más frecuentes en los fallecidos; mientras que las bandas parenquimales, distribución periférica de las lesiones y el patrón en parches fueron más frecuentes en los sobrevivientes. Todos estos hallazgos mostraron una diferencia significativa entre ambos grupos (Tabla 2).
Con relación a la extensión del compromiso pulmonar, se observó que el compromiso fue bilateral en 250 (98,4\%) casos, siendo más frecuente que los cinco lóbulos pulmonares se vieran comprometidos (94,5\%), y de ellos, el lóbulo inferior derecho (LID) con mayor frecuencia (61,4\%) (Anexo 3).

\section{Puntuación tomográfica de severidad (PTS)}

La aplicación de la PTS por ambos evaluadores mostró un análisis de repetibilidad adecuado con un CCI de 0,93 (IC 95\%: 0,85-0,97). La mediana del valor de la PTS en los 254 casos evaluados fue 13 (RIC: 11-15); en los sobrevivientes fue 12 (RIC: 9-14) y en los fallecidos 16 (RIC: 14-17), se observó una diferencia significativa entre ambos grupos, $\mathrm{p}<0,001(\mathrm{Ta}-$ bla 2). Con relación al grado de severidad clínica, la mediana del valor de la PTS en los casos moderados fue 11 (RIC: 9-13) $y$ en los casos severos fue 15 (RIC: 13-16) (Tabla 3).

El análisis de la curva ROC para los casos moderados mostró un área bajo la curva $(\mathrm{ABC})$ de 0,99 (IC 95\%: 0,98- 
Tabla 2. Hallazgos tomográficos en pacientes con la COVID-19, fallecidos y sobrevivientes atendidos en el Hospital Nacional Edgardo Rebagliati Martins, abril del 2020

\begin{tabular}{|c|c|c|c|c|}
\hline \multirow[t]{2}{*}{ Hallazgos tomográficos } & $\begin{array}{c}\text { Total } \\
\text { (254 casos) }\end{array}$ & $\begin{array}{l}\text { Fallecidos } \\
(95 \text { casos })\end{array}$ & $\begin{array}{c}\text { Sobrevivientes } \\
\text { (159 casos) }\end{array}$ & \multirow[t]{2}{*}{ Valor de $p$} \\
\hline & n (\%) & n (\%) & n (\%) & \\
\hline Opacidades en vidrio esmerilado & $243(95,7)$ & $94(99,0)$ & $149(93,7)$ & $0,047^{\mathrm{a}}$ \\
\hline Consolidaciones & $200(78,7)$ & $76(80,0)$ & $124(78,0)$ & $0,704^{\text {a }}$ \\
\hline «Preservación subpleural» & $184(72,4)$ & $77(81,1)$ & $107(67,3)$ & $0,018^{\text {a }}$ \\
\hline EII & $182(71,7)$ & $80(84,2)$ & $102(64,2)$ & $0,001^{\mathrm{a}}$ \\
\hline Broncograma aéreo & $120(47,2)$ & $47(49,5)$ & $73(45,9)$ & $0,582^{\text {a }}$ \\
\hline «Empedrado» & $90(35,4)$ & $59(62,1)$ & $31(19,5)$ & $<0,001^{\text {a }}$ \\
\hline Bandas parenquimales & $47(18,5)$ & $3(3,16)$ & $44(27,7)$ & $<0,001^{\mathrm{b}}$ \\
\hline Adenopatías & $15(5,9)$ & $10(10,5)$ & $5(3,1)$ & $0,025^{\mathrm{b}}$ \\
\hline Efusión pleural & $7(2,8)$ & $4(4,2)$ & $3(1,9)$ & $0,430^{\mathrm{b}}$ \\
\hline Signo del «halo reverso» & $5(2,0)$ & 0 & $5(3,1)$ & NC \\
\hline Distribución & & & & $<0,001^{\text {b }}$ \\
\hline Mixta & $199(78,3)$ & $86(90,5)$ & $113(71,1)$ & \\
\hline Periférica & $49(19,3)$ & $8(8,4)$ & $41(25,8)$ & \\
\hline Central & $6(2,4)$ & $1(1,1)$ & $5(3,1)$ & \\
\hline Patrón & & & & $<0,001^{\mathrm{a}}$ \\
\hline Difuso & $188(74,0)$ & $86(90,5)$ & $102(64,2)$ & \\
\hline En parches & $66(26,0)$ & $9(9,5)$ & $57(35,8)$ & \\
\hline PTS, mediana (RIC) & $13(11-15)$ & $16(14-17)$ & $12(9-14)$ & $<0,001^{\mathrm{c}}$ \\
\hline
\end{tabular}

RIC: rango intercuartílico, EII: engrosamiento intersticial interlobulillar, PTS: puntuación tomográfica de severidad, NC: no calculado.

${ }^{a}$ Prueba de Chi cuadrado, ${ }^{\mathrm{b}}$ prueba exacta de Fisher, ${ }^{\mathrm{c}}$ prueba de suma de rangos de Wilcoxon.

1,00) y un punto de corte de 7 presentó sensibilidad de $94,4 \%$ y especificidad de $100 \%$. La curva ROC para los casos severos mostró una $\mathrm{ABC}$ de 0,86 (IC 95\%: 0,82-0,90) y un punto de corte de 13 mostró sensibilidad de $84,9 \%$ y especificidad de 70,6\% (Anexo 4).

En la Figura 1 se presentan imágenes tomográficas de tres pacientes incluidos en el estudio, se describen los principales hallazgos identificados y el valor de la PTS asignado.

\section{Análisis multivariado, regresión de Cox y curva de sobrevida de Kaplan-Meier}

Los hallazgos tomográficos asociados a severidad clínica fueron el patrón difuso de las lesiones (OR: 3,23; IC 95\%: 1,46-7,14), patrón en «empedrado» (OR: 2,48; IC 95\%: 1,08-5,68) y un mayor valor en la PTS (OR: 1,73; IC 95\%: 1,49-2,02) (Tabla 3).

Los hallazgos tomográficos asociados a mayor mortalidad en pacientes severos en el análisis de sobrevida me-
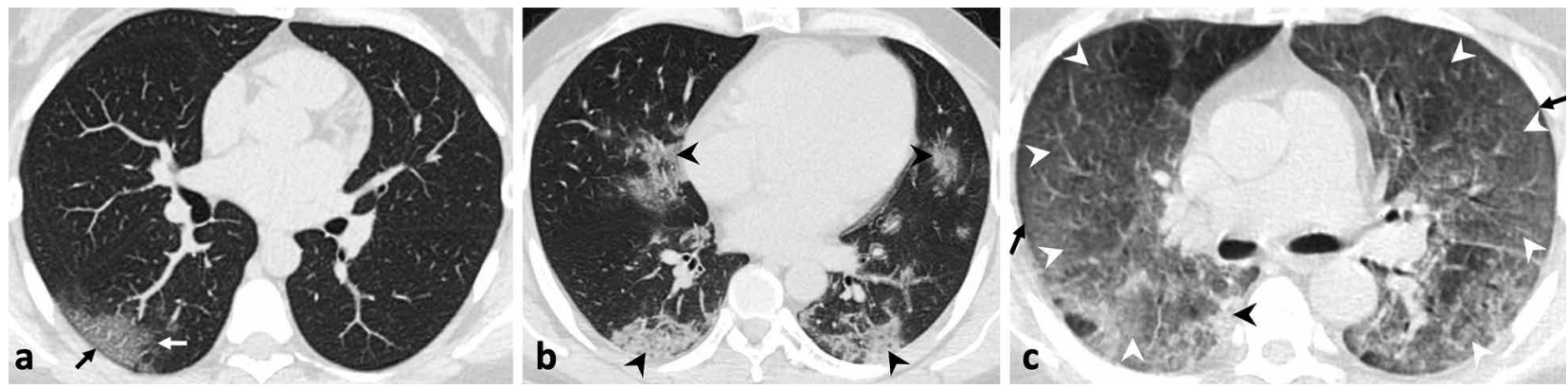

Figura 1. Tomografía pulmonar sin contraste en vista axial (a, b, c). a) Mujer de 31 años con fiebre, malestar general y diarrea. Se observan opacidades en vidrio esmerilado (flecha blanca) de distribución periférica, y el signo de «preservación subpleural» (flecha negra), puntuación tomográfica de severidad (PTS): 2. b) Varón de 45 años con fiebre, tos, disnea, diarrea y dolor torácico. Se observan consolidaciones (cabezas de flechas negras) de distribución central y periférica, PTS: 9. c) Varón de 45 años con fiebre y disnea. Se observan extensas áreas de patrón en «empedrado» (cabezas de flechas blancas) con patrón difuso que compromete ambos pulmones, así como el signo de «preservación subpleural» (flecha negra) y consolidación (cabeza de flecha negra), PTS: 19. 
Tabla 3. Hallazgos tomográficos asociados a casos severos en pacientes con la COVID-19 atendidos en el Hospital Nacional Edgardo Rebagliati Martins, abril del 2020.

\begin{tabular}{|c|c|c|c|c|}
\hline Hallazgos tomográficos & $\begin{array}{c}\text { Leve y moderado } \\
(102 \text { casos }) \\
\mathbf{n}(\%)\end{array}$ & $\begin{array}{c}\text { Severo } \\
(152 \text { casos }) \\
\text { n }(\%)\end{array}$ & ORc (IC 95\%) & ORa (IC 95\%) \\
\hline Consolidaciones & $76(74,5)$ & $124(81,6)$ & $1,51(0,83-2,75)$ & $1,02(0,41-2,50)$ \\
\hline «Preservación subpleural» & $63(61,8)$ & $121(79,6)$ & $2,42(1,38-4,24)$ & $1,79(0,87-3,69)$ \\
\hline EII & $58(56,9)$ & $124(81,6)$ & $3,36(1,91-5,92)$ & $1,81(0,83-3,94)$ \\
\hline Broncograma aéreo & $38(37,3)$ & $82(53,9)$ & $1,97(1,18-3,29)$ & $1,68(0,82-3,45)$ \\
\hline «Empedrado» & $16(15,7)$ & $74(48,7)$ & $5,10(2,74-9,50)$ & $2,48(1,08-5,68)$ \\
\hline Bandas parenquimales & $33(32,4)$ & $14(9,2)$ & $0,21(0,10-0,42)$ & $0,34(0,14-0,75)$ \\
\hline \multicolumn{5}{|l|}{ Distribución } \\
\hline Periférica & $34(33,3)$ & $15(9,9)$ & $0,21(0,11-0,42)$ & $0,57(0,24-1,34)$ \\
\hline Central & $3(3,0)$ & $3(2,0)$ & $0,49(0,10-2,47)$ & $0,92(0,14-6,07)$ \\
\hline Mixta & $65(63,7)$ & $134(88,2)$ & 1 & 1 \\
\hline \multicolumn{5}{|l|}{ Patrón } \\
\hline Difuso & $54(52,9)$ & $134(88,2)$ & $6,62(3,53-12,39)$ & $3,23(1,46-7,14)$ \\
\hline En parches & $48(47,1)$ & $18(11,8)$ & 1 & 1 \\
\hline PTS & $10(8-13)^{a}$ & $15(13-16)^{\text {a }}$ & $1,76(1,52-2,03)^{b}$ & $1,73(1,49-2,02)^{b}$ \\
\hline
\end{tabular}

EII: engrosamiento intersticial interlobulillar, PTS: puntuación tomográfica de severidad. ORc: Odds ratio bivariado crudo (cada hallazgo tomográfico de forma independiente).

ORa: Odds ratio multivariado (todos los hallazgos tomográficos + covariables)

${ }^{\mathrm{a}}$ mediana (rango intercuartílico), ${ }^{\mathrm{b}}$ PTS + covariables.

Covariables: edad, sexo, obesidad, tiempo de enfermedad, hipertensión arterial y diabetes.

diante regresión de Cox fueron la presencia de patrón en «empedrado» (HR: 1,78; IC 95\%: 1,03-3,06) y mayor valor en la PTS (HR: 1,33; IC 95\%: 1,20-1,48) (Tabla 4). Además, se identificó que la sobrevida en pacientes severos, tras 86

Tabla 4. Hallazgos tomográficos asociados a mortalidad en casos severos de pacientes con la COVID-19 atendidos en el Hospital Nacional Edgardo Rebagliati Martins, abril del 2020.

\begin{tabular}{lcc}
\hline Hallazgos tomográficos & HRc $(\mathbf{I C}$ 95\%) & HRa (IC 95\%) \\
\hline «Preservación subpleural» & $0,84(0,50-1,42)$ & $0,85(0,50-1,48)$ \\
EII & $0,66(0,33-1,30)$ & $0,67(0,34-1,34)$ \\
«Empedrado» & $2,35(1,40-3,93)$ & $1,78(1,03-3,06)$ \\
Distribución & & \\
$\quad$ Mixta & 1 & 1 \\
$\quad$ Periférica & $0,68(0,31-1,51)$ & $0,72(0,31-1,68)$ \\
$\quad$ Central & $1,11(0,15-8,38)$ & $1,34(0,17-10,34)$ \\
Patrón & & \\
$\quad$ Difuso & $1,22(0,57-2,61)$ & $1,23(0,54-2,80)$ \\
PTS & & \\
$\quad$ Continuo & $1,33(1,19-1,47)^{\text {a }}$ & $1,33(1,20-1,48)^{\mathrm{a}}$ \\
\hline
\end{tabular}

EII: engrosamiento intersticial interlobulillar, PTS: puntuación tomográfica de severidad.

HRc: Hazard ratio crudo.

HRa: Hazard ratio multivariado (todos los hallazgos tomográficos + covariables) a PTS + covariables.

Covariables: edad, sexo, obesidad, tiempo de enfermedad, hipertensión arterial y diabetes. días de seguimiento, fue menor en aquellos con valores de PTS $\geq 14$ respecto a aquellos con valores de PTS $<14$. Este resultado se grafica en la curva de Kaplan Meier presentada en la Figura 2.

\section{DISCUSIÓN}

Los hallazgos tomográficos más frecuentes fueron las opacidades en vidrio esmerilado y las consolidaciones; los menos frecuentes fueron las adenopatías y efusión pleural. Los ha-

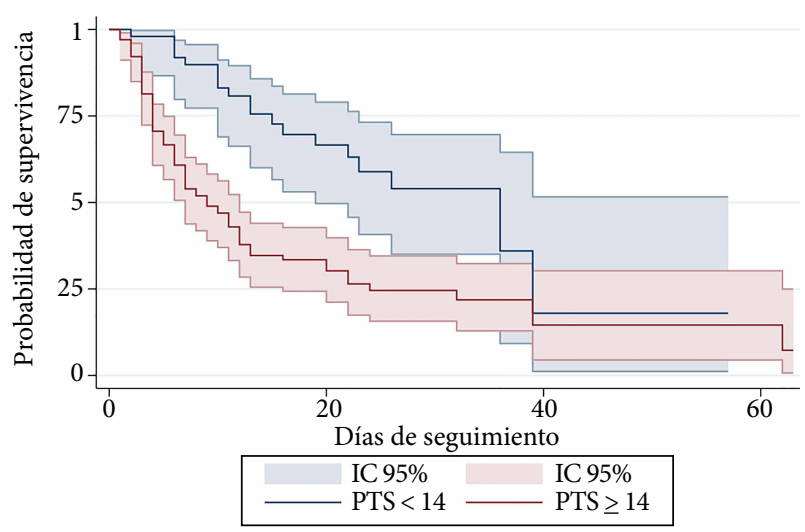

Figura 2. Curva de supervivencia de Kaplan-Meier. Comparación de las tasas de supervivencia estimadas en casos severos con puntuación tomográfica de severidad (PTS) $<14$ y casos severos con PTS $\geq 14$. 
llazgos asociados a severidad clínica fueron el patrón difuso de las lesiones, patrón en «empedrado» y mayor valor de la PTS; mientras que los hallazgos asociados a mortalidad en casos graves fueron el patrón en «empedrado» y un mayor valor de la PTS. Un valor de la PTS de 7 mostró sensibilidad de $94,4 \%$ y especificidad de $100 \%$ para identificar casos moderados, y un valor de 13 mostró sensibilidad de 84,9\% y especificidad de $70,6 \%$ para identificar casos severos.

Entre las variables demográficas y clínicas evaluadas, la diabetes mellitus y obesidad no mostraron diferencia significativa entre sobrevivientes y fallecidos, contrario a lo descrito en reportes internacionales ${ }^{(23-26)}$. Este hallazgo podría explicarse por un subregistro de estas comorbilidades en las historias clínicas de los pacientes durante su evaluación inicial en emergencia.

La mayor frecuencia de opacidades en vidrio esmerilado y consolidaciones coincide con lo descrito por múltiples estudios realizados en otros países desde el inicio de la pandemia ${ }^{(6-10)}$. El signo del «halo reverso» fue un hallazgo infrecuente $(2,0 \%)$, similar a lo descrito por Bernheim ${ }^{(12)}$ pero contrario al $10 \%$ reportado por Tabatabaei et al. ${ }^{(27)}$. El signo de «preservación subpleural» se observó con bastante frecuencia $(72,4 \%)$ en este estudio; se trata de un signo descrito clásicamente en neumonía intersticial no específica, contusión pulmonar y otras infecciones virales como influenza, pero que también ha sido reportado por algunos autores ${ }^{(15,28)}$ en casos de neumonía por la COVID-19.

La distribución mixta (central y periférica) y el patrón difuso de las lesiones observado en la mayoría de los casos, podría explicarse por el mayor número de pacientes con enfermedad moderada o severa incluidos en el estudio. De forma contraria, los casos con enfermedad leve mostraron lesiones de distribución principalmente periférica y patrón en parches. En relación con el compromiso de los lóbulos pulmonares, el LID fue el lóbulo afectado con mayor frecuencia $(61,4 \%)$, en contraste a lo reportado por Long et al. ${ }^{(8)}$, quienes encontraron que se afectaba el LII en la mayoría de los casos $(66,7 \%)$.

Con relación a la PTS, el estudio publicado por Li et al. ${ }^{(16)}$ propuso un valor de 7,5 para identificar los casos de enfermedad severa-crítica con una sensibilidad de $82,6 \%$ y especificidad de $100 \%$. En nuestro país se utiliza una clasificación de severidad clínica distinta: casos leves, moderados y severos ${ }^{(21)}$; por lo que este estudio determinó un valor de 7 de la PTS para identificar a los casos moderados y 13 para los casos severos, ambos con una adecuada sensibilidad y especificidad.

Los hallazgos tomográficos asociados a mayor severidad y mortalidad, como el patrón difuso de las lesiones y un mayor valor de la PTS, coinciden con lo descrito por otros estudios. Estos mencionan que una mayor extensión del compromiso pulmonar, valorada mediante distintas puntuaciones tomográficas, se relaciona con mayor probabilidad de ingreso a la UCI y a mayor mortalidad ${ }^{(16.17)}$. Asimismo, el patrón en «empedrado» también ha sido asociado a cuadros clínicos más severos en algunos estudios ${ }^{(20,27)}$. Se menciona que el análisis de los hallazgos tomográficos asociados a mortalidad se realizó solo en el grupo de pacientes severos debido a que casi todos los fallecidos $(98,9 \%)$ correspondían a este grupo de pacientes.

No se encontró asociación entre la presencia de consolidaciones y el grado de severidad clínica o riesgo de mortalidad, contrario a lo reportado por autores como Li et al. ${ }^{(29)}$ pero similar a lo encontrado por Zhou et al. ${ }^{(24)}$; probablemente debido a que, según la progresión de los hallazgos tomográficos descritos por Pan et al ${ }^{(18)}$, la presencia de consolidaciones dependería más del tiempo de evolución de la enfermedad, que de la severidad del cuadro clínico.

Entre las limitaciones del estudio se encuentra el reducido número de pacientes incluidos con enfermedad leve, esto debido a que el estudio se llevó a cabo en una institución de referencia nacional, la cual recibe una mayor proporción de casos moderados y severos. Sin embargo, se debe tener en cuenta que la utilidad de la TC de tórax es mayor en este grupo de pacientes, y mínima en pacientes con enfermedad leve. Además, se incluyeron a pacientes con prueba serológica positiva debido a que este criterio formaba parte de los lineamientos del MINSA para el diagnóstico de la COVID-19, considerando también que todos los pacientes incluidos presentaban cuadro clínico y representación tomográfica de esta enfermedad. Por último, no se incluyó entre las variables estudiadas el tratamiento recibido por los pacientes, debido a que la determinación de severidad se realizó en base a la evaluación inicial registrada en emergencia, y a que no se contaba con un esquema de referencia para el tratamiento de esta enfermedad al momento del estudio.

En conclusión, el patrón difuso de las lesiones se asoció a mayor severidad clínica. El patrón en «empedrado» y un mayor valor en la PTS se asociaron a mayor severidad clínica y también a mortalidad. Se proponen valores de 7 y 13 como puntos de corte de la PTS para identificar casos moderados y severos de la COVID-19.

Se recomienda usar los resultados obtenidos en el presente estudio con el fin de complementar la evaluación de los pacientes con la COVID-19 en el ámbito hospitalario. Se sugiere también desarrollar más estudios en nuestro medio que evalúen la relación de los hallazgos tomográficos pulmonares con la mortalidad, incluidos los tratamientos que hasta el momento han demostrado influir en ella.

Agradecimientos: Los autores agradecen a la Dra. Malú Arias Schreiber, al Dr. Johnni Huamán y al Dr. Henry Tito por su valoración crítica a este estudio, así como a todo el personal de salud del Hospital Nacional Edgardo Rebagliati Martins que trabaja en primera línea atendiendo a los pacientes afectados por la COVID-19.

Contribución de los autores: JCG, VPB, RCA y MM participaron en la concepción del estudio, recolección de datos, análisis e 
interpretación de resultados. Todos los autores participaron en la redacción del manuscrito, revisión crítica y aprobación de la versión final.

Financiamiento: Autofinanciado.

\section{REFERENCIAS BIBLIOGRÁFICAS}

1. Huang C, Wang Y, Li X, Ren L, Zhao J, Hu Y, et al. Clinical features of patients infected with 2019 novel coronavirus in Wuhan, China. Lancet. 2020;395(10223):497-506. doi: 10.1016/S0140-6736(20)30183-5.

2. Zhu N, Zhang D, Wang W, Li X, Yang B, Song J, et al. A Novel Coronavirus from Patients with Pneumonia in China, 2019. N Engl J Med. 2020;382:727-33. doi: 10.1056/NEJMoa2001017.

3. Organización Mundial de la Salud [Internet]. Ginebra: OMS; 2020 [citado el 29 de abril del 2020]. Discursos del Director General de la OMS. Disponible en: https://www.who.int/es/dg/speeches/detail/ who-director-general-s-remarks-at-the-media-briefing-on-2019-ncovon-11-february-2020.

4. Organización Mundial de la Salud [Internet]. Ginebra: OMS; 2020 [citado el 29 de abril del 2020]. La OMS caracteriza a COVID-19 como una pandemia; Disponible en: https://www.paho.org/es/noticias/11-3-2020-oms-caracteriza-covid-19-como-pandemia.

5. Rubin GD, Ryerson CJ, Haramati LB, Sverzellati N, Kanne JP, Raoof $\mathrm{S}$, et al. The Role of Chest Imaging in Patient Management during the COVID-19 Pandemic: A Multinational Consensus Statement from the Fleischner Society. Radiology. 2020;296(1):172-180. doi: 10.1148/ radiol.2020201365.

6. Ai T, Yang Z, Hou H, Zhan C, Chen C, Lv W, et al. Correlation of Chest CT and RT-PCR Testing in Coronavirus Disease 2019 (COVID-19) in China: A Report of 1014 Cases. Radiology. 2020;296(2):E32-E40. doi: 10.1148/radiol.2020200642.

7. Xie X, Zhong Z, Zhao W, Zheng C, Wang F, Liu J. Chest CT for Typical 2019-nCoV Pneumonia: Relationship to Negative RT-PCR Testing. Radiology. 2020;296(2):E41-E45. doi: 10.1148/radiol.2020200343.

8. Long C, Xu H, Shen Q, Zhang X, Fan B, Wang C, et al. Diagnosis of the Coronavirus disease (COVID-19): rRT-PCR or CT?. Eur J Radiol. 2020;126:108961. doi: 10.1016/j.ejrad.2020.108961.

9. Caruso D, Zerunian M, Polici M, Pucciarelli F, Polidori T, Rucci C, et al. Chest CT Features of COVID-19 in Rome, Italy. Radiology. 2020;296(2):E79-E85. doi: 10.1148/radiol.2020201237.

10. Fang $\mathrm{Y}$, Zhang H, Xie J, Lin M, Ying L, Pang P, et al. Sensitivity of Chest CT for COVID-19: Comparison to RT-PCR. Radiology. 2020;296(2):E115-E117. doi: 10.1148/radiol.2020200432.

11. Kim H, Hong H, Yoon SH. Diagnostic Performance of CT and Reverse Transcriptase-Polymerase Chain Reaction for Coronavirus Disease 2019: A Meta-Analysis. Radiology. 2020. doi: 10.1148/radiol.2020201343.

12. Bernheim A, Mei X, Huang M, Yang Y, Fayad ZA, Zhang N, et al. Chest CT Findings in Coronavirus Disease-19(COVID-19): Relationship to Duration of Infection. Radiology. 2020;295(3). doi: 10.1148/radiol.2020200463.

13. Ding X, Xu J, Zhou J, Long Q. Chest CT findings of COVID-19 pneumonia by duration of symptoms. Eur J Radiol. 2020;127. doi: 10.1016/j. ejrad.2020.109009.

14. Chung M, Bernheim A, Mei X, Zhang N, Huang M, Zeng X, et al. CT Imaging Features of 2019 Novel Coronavirus (2019-nCoV). Radiology. 2020;295(1):202-7. doi: 10.1148/radiol.2020200230.

15. Prokop M, van Everdingen W, van Rees Vellinga T, Quarles van Ufford J, Stöger L, Beenen L, et al. CO-RADS - A categorical CT assessment scheme for patients with suspected COVID-19: definition and evaluation. Radiology. 2020;296(2):E97-E104. doi: 10.1148/radiol.2020201473.
Conflictos de interés: Los autores niegan tener algún conflicto de interés que pueda afectar la objetividad del estudio.

Material suplementario: Disponible en la versión electrónica de la RPMESP

16. Li K, Fang Y, Li W, Pan C, Qin P, Zhong Y, et al. CT image visual quantitative evaluation and clinical classification of coronavirus disease (COVID-19). Eur Radiol. 2020;30:4407-4416. doi: 10.1007/s00330-020 06817-6.

17. Francone M, Iafrate F, Masci GM, Coco S, Cilia F, Manganaro L, et al. Chest CT score in COVID-19 patients: correlation with disease severity and short-term prognosis. Eur Radiol 2020. doi: 10.1007/s00330-020-07033-y.

18. Pan F, Ye T, Sun P, Gui S, Liang B, Li L, et al. Time Course of Lung Changes at Chest CT during Recovery from Coronavirus Disease 2019(COVID-19). Radiology. 2020;295(3):715-721. doi: 10.1148/radiol.2020200370.

19. Yang R, Li X, Liu H, Zhen Y, Zhang X, Xiong Q, et al. Chest CT Severity Score: An Imaging Tool for Assessing Severe COVID-19. Radiol Cardiothorac Imaging 2020;2(2):e200047. doi: 10.1148/ryct.2020200047.

20. Zhang R, Ouyang H, Fu L, Wang S, Han J, Huang K, et al. CT features of SARS-CoV-2 pneumonia according to clinical presentation: a retrospective analysis of 120 consecutive patients from Wuhan city. Eur Radiol. 2020;30(8):4417-4426. doi: 10.1007/s00330-020-06854-1.

21. Ministerio de Salud. Documento técnico Prevención Diagnóstico y Tratamiento de personas afectadas por COVID-19 en el Perú [Internet]. Lima: MINSA; 2020 [Citado el 29 de abril del 2020]. Disponible en: https://www. gob.pe/institucion/minsa/informes-publicaciones/473587-prevencion-diagnostico-y-tratamiento-de-personas-afectadas-por-covid-19-en-el-peru.

22. Hansell DM, Bankier AA, MacMahon H, McLoud TC, Müller NL, Remy J. Fleischner Society: Glossary of Terms for Thoracic Imaging. Radiology. 2008;246(3):697-722. doi: 10.1148/radiol.2462070712.

23. Li X, Xu S, Yu M, Wang K, Tao Y, Zhou Y, et al. Risk factors for severity and mortality in adult COVID-19 inpatients in Wuhan. Journal of Allergy and Clinical Immunology. 2020;146(1)110-118. doi: 10.1016/j. jaci.2020.04.006.

24. Zhou F, Yu T, Du R, Fan G, Liu Y, Liu Z, et al. Clinical course and risk factors for mortality of adult inpatients with COVID-19 in Wuhan, China: a retrospective cohort study. The Lancet. 2020;395(10229):1054-1062. doi: 10.1016/S0140-6736(20)30566-3.

25. Albitar O, Ballouze R, Ooi JP, Ghadzi SMS. Risk factors for mortality among COVID-19 patients. Diabetes Research and Clinical Practice. 2020;166:e108293. doi: 10.1016/j.diabres.2020.108293.

26. Mikami T, Miyashita H, Yamada T, Harrington M, Steinberg D, Dunn A et al. Risk Factors for Mortality in Patients with COVID-19 in New York City. J Gen Intern Med. 2021;36:17-26. doi: 10.1007/s11606-020-05983-z.

27. Tabatabaei SMH, Talari H, Moghaddas F, Rajebi H. Computed Tomographic Features and Short-term Prognosis of Coronavirus Disease 2019 (COVID-19) Pneumonia: A Single-Center Study from Kashan, Iran. Radiol Cardiothorac Imaging. 2020;2(2):e200130. doi: 10.1148\%2Fryct.2020200130.

28. Wu J, Pan J, Teng D, Xu X, Feng J, Chen Y. Interpretation of CT signs of 2019 novel coronavirus (COVID-19) pneumonia. Eur Radiol. 2020;30(10):5455-5462. doi: 10.1007/s00330-020-06915-5.

29. Li K, Wu J, Wu F, Guo D, Chen L, Fang Z, et al. The Clinical and Chest CT Features Associated With Severe and Critical COVID-19 Pneumonia. Investive Radiology. 2020;55(6):327-331. doi: 10.1097/ rli.0000000000000672. 